\title{
Cryphonectria parasitica (Murr) Barr EN LAS COMUNIDADES DE MICROHONGOS EPIFITOS DE LA CORTEZA DEL CASTAÑO (Castanea sativa Miller) EN EL NORTE DE ITALIA
}

Cryphonectria parasitica (Murr) Barr in the epiphytic microfungal communities of the chestnut bark (Castanea sativa Miller) in northern Italy

\author{
Anna Maria Picco* \\ Edoardo Piontelli, L. ** \& Stefania Bottaso* \\ *Universitá degli Studi di Pavia , Facoltá di Scienze Mat. Fis. Nat.Istitutod \\ Micologia Medica "R.Ciferri e P. Redaelli"Via S.Epifanio 14, 27100 Pavia, Italia. \\ **Universidad de Valparaiso, Escuela de Medicina, \\ Cátedra de Micología, Casilla 92 V, Valparaiso, Chile
}

Palabras clave: Comunidades fúngicas, ecología, biodiversidad,cancro de la corteza del castaño, Cryphonectria parasitica

Key words : Fungal community,ecology,biodiversity, chestnut blight, Cryphonectria parasitica.

\section{RESUMEN}

Mediante la incubación en agar agua, de trozos de corteza del tronco de 7 grupos de castaños (Castanea sativa Miller), en una localidad del norte de Italia, se aislaron 780 cepas de microhongos filamentosos repartidos en 78 taxa. Se consideraron diferentes variables, tales como: presencia (C/C) o ausencia de cancro (S/C), tipos de corteza yedad de los árboles. Los mayores aislamientos fueron en la corteza del tronco y dela base de los árboles viejos (67\%) y jóvenes $(64,8 \%) C / C$. La cantidad de patógenos potenciales fue levemente mayor en la corteza del tronco de los árboles $C / C$.

Las comunidades deDeuteromycetes fueron las más representativas y dentro de estas los Hyphomycetes dematiaceos, los más diversificados. Sólo el 10,5\% del total de los taxa se presentaron en todas las combinaciones estudiadas: Acremonium strictum, Alternaria alternata, Ceratocystis microspora, Cryphonectria parasitica, Penicillium spp., Sporidesmium rubi y Trichoderrma spp.

Cryphonectria parasitica, Ceratocystis microspora y Ophiostoma stenocerans, mantienen con Cr.parasitica una estrecha asociación ya sea en los árboles $C / C$ como en los $S / C$, en todas las situaciones.

Las más altas diversidades (Shannon-Wieber) se observaron en árboles C/C y las mayores similitudes (Jaccard) entre los árboles $S / C$, en corteza tronco y entre esta y la corteza base.

\section{SUMMARY}

By incubating some pieces of bark from the trunk of 7 groups of chestnut trees in water agar, in a locality of northern Italy, 780 strains of filamentous microfungi spread in 78 taxa were isolated. Different variables such as :presence $(C / C)$ or absence of blight $(S / C)$, tipes of bark and age of trees were considered.

Highest isolations were found in the bark of the trunk and in the base of old (67\%) and young (64,8\%) C/C) trees. The number of potential pathogenics was slightly higher in the bark of trunks from $C / C$ trees. Deuteromycetes communities were the most representative and within the former Dematiaceous Hyphomycetes showed greater diversity. Only 10,5\% of the total taxa were present in every combination examined: Acremonium strictum, Alternaria alternata, Ceratocystis microspora, Cryphonectria parasitica, Penicillium spp., Sporidesmium rubi and Trichoderrma spp.

Cryphonectria parasitica, Ceratocystis microspora and Ophiostoma stenocerans keep a close relation with $\mathrm{Cr}$. parasitica, both in $C / C$ and $S / C$ trees under every conditions.

Highest diversities (Shannon-Wieber) were observed in $C / C$ trees, while the highest simitudes (Jaccard) were found in $S / C$ trees in the bark of the trunk and between the latter and the base bark. 


\section{INTRODUCCION}

El castaño (Castanea sativa Miller), es un árbol ampliamente difundido en las áreas temperadas septentrionales de Eurasia, America Nord-Oriental y las zonas temperadas de Sud America. (Anagnostakis, 1990; Bassi, 1990; Gobbi\&Locci,1989). Suamplia distribucióngeográfica se debe principalmente a la acción del hombre, por sus características botánicas, ecológicas, paisajísticas y económicas.

Su cultivo en Italia, como en otros paises europeos, ha disminuido notablemente, debido al uso industrial indiscriminado de su madera, al abandono de sus frutos como fuente de alimento fundamental, a la disminución de mano de obra en los campos por la migración campesina al área industrial. Pero principalmente por diversas enfermedades producidas por hongos, ya sea en sus raices, troncos y hojas (Anagnostakis, 1987; Cobos, 1989), oel ataque deLepidopteros y Coleopteros (Mansilla, 1984).

Los graves daños causados en USA y Europa por el cancro de su corteza por Cryphonectria parasitica $(=E n-$ dothia parasitica Murr.), han diezmado sus poblaciones hasta casi el exterminio (Hepting 1974).

En Italia fue detectado en 1938 en la Liguria, cerca de Genova, extendiendose rapidamente a toda la península (Biraghi, 1950; Goidanich 1960; Anagnostakis, 1987; Turchetti et al. 1991,1992,) y a otros paises de la comunidad europea (Conedera,1991; Muñoz\& Cobos, 1991; Gobbi\&Locci,1989). En la actualidad debido a los daños, se ha volcado el interés científico hacia su control biológico mediante especies hipovirulentas de este hongo $\mathrm{u}$ otros microorganismos (Russin \& Shain,1984; Anagnostakis, 1987; Turchetti \& Maresi, 1991; Wilhelm, 1992; Minervini \&Bisiach, 1994).

La presencia, biomasa, distribución y los posibles efectos antagónicos de algunos microhongos patógenos o saprotrofos en ambientes definidos, pueden modificar el equilibrio de un particular habitat, repercutiendo en la biodiversidad total de la comunidad presente (Wells \& Paine, 1975; Russin \& Shain, 1984; Sung \& Han, 1986; Baird, 1991; Minervini \& Bisiach. 1994; Behrend et al. 1994).

Son pocos los estudios efectuados en diversas paises y en Italia, para conocer los hongos que viven asociados a las especies de Castanea, que aporten datos epidemiológicos, diversidad de especies, distribución, frecuencia de patrones de colonización, posibles interacciones o asociaciones y la producción de metabolitos tóxicos (Sung \& Han, 1986; Turchetti, 1986; Cobos 1989; Baird, 1991; Minervini\& Bisaghi, 1994; Bisseger \& Sieber, 1994).

El objetivo de nuestro trabajo consistió en : estudiar la presencia de las comunidades de microhongos filamentosos epífitos (saprotrofos, parásitos y oportunistas), asociados a
Cryphonectria parasitica, en la corteza del castaño (tronco y base), en un período estacional cálido, según grupos etáreo y presencia de cancro.

\section{MATERIALESY METODOS}

Esta investigación se efectuó en la localidad de Giovo Ligure (Provincia de Savona) entre mayo y octubre1993, considerando como un todo un área de 7 zonas distantes pocos kilómetros entre si, con castaños de diferentes edades. Los terrenos tenían altitudes s.n.m, que oscilaban entre 490 a $550 \mathrm{~m}$ y una pluviosidad anual cercana a los $1000 \mathrm{~mm}$.

\section{1) Características y ubicación de las zonas.}

A cada zona muestreada se le asignó una letra, de la A a la F. La zona A, E1, E2 y F (Grupo 1) estaba integrada por castaños cuyas edades finctuaban entre los 15 a 30 años, mientras las zonas B, C y D (Grupo 2) por castaños entre 60 a 80 años.

a)En el Grupo 1, las localidades geográficas fueron :

A (Colle di Giovo), con una tipología boscosa constituida primariamente por Castanea sativa, con presencia esporádicade Robiniapseudoacacia.L.y Quercuspubescens Will.. Terreno de elevada pendiente, recubierto por gramíneas diversas

$\mathbb{E} 1$ (La Barchina, sur-oeste), tipología boscosa constituida por Castanea sativa al estado silvestre. Terreno con alta pendiente, fuertemente recubierto de gramíneas y arbustos no infestantes.

$\mathbb{E} 2$ (La Barchina, oeste), tipología boscosa y terrenos identicos a $\mathrm{E} 1$.

F (Cercanías del rio Maglialunga), tipología boscosa mixta constituida por Castanea sativa y helechos. Terreno con pendiente media, recubierto por gramíneas diversas y arbustos no infestantes.

b) En el Grupo 2, las localidades geográficas fueron:

$\mathbb{B}$ ( Colle il Giardino), tipología boscosa constituida solamente por Castanea sativa (probablemente representa residuos de un viejo bosque de castaños productivos). Terreno practicamente plano, muy limpio, recubierto de pastizales que se cortan periodicamente por sus dueños, para forraje animal.

C (Rezeché), tipología boscosa dispersa constituida por Castanea sativa de diferentes edades. Terreno con pendiente intermedia, muy limpio y con similares mantenimientos al igual que $\mathbf{B}$.

D (Bricco Mondo), tipología boscosa constitiuda por un pequeño grupo de viejos castaños ( $\boldsymbol{C}$. sativa ). Terreno con pendiente intermedia, muy limpio al igual que $\mathbf{B}$ y $\mathbf{C}$. 


\section{2) Técnicas de muestreo.}

Se efectuaron 2 muestreos en cada grupo, en un período comprendido entre primavera y finales de verano, siendo el lapso entre el primer y segundo de 3 meses. Cada muestreo abarcó un período de 1 mes y se efectuó considerando las características siguientes:

a) presencia de cancros visibles en la corteza del tronco

b) presencia de corteza aparentemente sana del tronco

c) corteza del tronco a una altura entre 1 a 2,5 m

d) corteza del tronco en la zona del cuello entre 10 a $50 \mathrm{~cm}$ del nivel del suelo (Corteza base).

Encada unadelas 7 zonas geográficas seseleccionaron 8 castaños ( 4 con cancro visible y 4 aparentemente sanos), disponiendo el muestreo en cada uno de los árboles seleccionados de la siguiente manera:

- Extracción de 3 trozos de corteza del tronco de unos $4-7 \times 2-4 \mathrm{~cm}$. app y 3 trozos de la corteza en la zona de la base, desde 4 árboles con cancro visibles del Grupo 1 (por 4 zonas) y Grupo 2 (por 3 zonas).

- Extracción de 3 trozos de la corteza del tronco y 3 de la base desde 4 árboles aparentemente sanos del Grupo 1 y 2 .

Los 3 trozos de cada muestra seleccionada según las variables se consideraba como un solo pool.

Los árboles seleccionados para el muestreo se marcaron con pintura blanca para poder reconocerlos para el segundo muestreo. El primer muestreo general (así como el segundo) arrojó un total de 32 pool de muestras en la corteza del tronco ( 16 con cancro y 16 sin cancro) y 32 en la corteza dela zonadelabaseenel Grupo 1.Enel Grupo2, 24 enlacorteza del tronco ( 12 con cancro y 12 sin cancro) y 24 en la corteza base. Total 112 pool de muestras.

La corteza se cortó con una herramienta metálica con filo(que se esterilizó a la llama de un pequeñomechero portátil a gas ) y los pool de cada variable se guardaron en bolsitas de plástico estériles que se refrigeraron $\left(-2^{\circ} \mathrm{C}\right)$ hasta su procesamiento (no superior a las 48 horas).

De este modo, para el estudio de los microhongos identificamos los 6 siguientes sustratos :

Arbolesjovenes(J), Viejos(V), Sin Cancro(S/C), Con Cancro (C/C), Corteza Base (C/B) y Corteza Tronco (C/T).

\section{Metodología decultivo}

Para que los hongos presentes pudieran desarrollarse solo en su sustrato natural, empleamos la técnica de la cámara húmeda en placas de Petri de $15 \mathrm{~cm}$, con agar agua ( $15 \mathrm{gr}$. de agaren $1000 \mathrm{ml}$ de agua). Cada pool demuestras decorteza, fue depositado sobre la superficie del agar (3 trozos por placa, lo que representaba 1 sola muestra). Las placas se incubaron a $25^{\circ} \mathrm{C}$ durante los primeros 15 días y luego a temperatura ambientehasta completar los 60 días (la temperaturamedia del laboratorio osciló entre los $19 \mathrm{a} 27^{\circ} \mathrm{C}$ ).

El crecimiento de estructuras, cuerpos fructiferos o colonias fúngicas, se controló mediante lupa estereoscópica a partir de los 2 días iniciales de incubación (para comprobar la presencia de cuerpos fructíferos maduros, desde el inicio del muestreo) y luego cada 10 días.

A pesar que la esporulación de la mayoría de los hongos que se desarrollaron sobre la corteza fue óptima y permitía una buena observación a la lupa estereoscópica, cuando fueron necesarios estudios complementarios, se efectuaron subcultivos en Agar Papa Dextrosay Agar Malta.

Las mediciones de las estructuras fúngicas se hicieron mediante preparaciones entre lámina y laminilla, utilizando como medio de montaje agua destilada, lactofenol solo o lactofenol con azul de algodón.

La presencia de una determinada especie o género en los 3 trozos de corteza de cada pool, se contabilizó una sola vez, aunque sus integrantes se presentaran repetidamente en la misma muestra.

No se consideró el tiempo de aparición de los hongos en la cámara húmeda (en los 60 días de observación) ni la diferencia estacional entre los muestreos. De esta manera los resultados de los 2 registros se sumaron en un solo total.

Se analizaron las comunidades de microhongos mediante el índice de diversidad de Shannon-Wieber en cada uno de los sustratos estudiados y el de similitud de Jaccard entre los sustratos.

\section{RESULTADOS}

Los resultados obtenidos permiten evidenciar la presencia de varios tipos de patrones de colonización fungica de la corteza del castaño en los sustratos estudiados. En la investigación se detectaron un total de 780 microhongos epífitos repartidos en 78 taxa. Los mayores aislamientos se produjeron en la corteza del troncoy de la base de los árboles jovenes $(64,8 \%$ del total ) y viejos $(67,5 \%) \mathrm{C} / \mathrm{C}$, mientras los menores en los S/C (J y V) (Gráfico 1). Semejante comportamiento se observó en la distribución de los géneros (Gráfico 2), sin embargo en la $\mathrm{C} / \mathrm{B}$, esas diferencias de taxa fúngicos fueron menores en $\mathrm{C} / \mathrm{T}$ que en $\mathrm{C} / \mathrm{B}$. El 56,3\% correspondió a $\mathrm{C} / \mathrm{B}$ y el resto de la $\mathrm{C} / \mathrm{T}$.

La distribución de géneros y especies consideradas patógenas u oportunistas en comparación con las saprotrofas, fuelevementemayor enla $\mathrm{C} / \mathrm{T}$ en los árboles $\mathrm{C} / \mathrm{C}(48.4$ y $46.5 \%$ del total) (Gráfico 3).

Al comparar el $100 \%$ del comportamiento de las 4 principales especies de patógenos u oportunistas (levemente superiores o inferiores al $25 \%$ del total de la micota aislada) en los 6 sustratos considerados, pudo apreciarse que el poo!, Cryphonectriaparasiticay Ceratocistis-Ophiostoma, mantienen una estrecha asociación ya sea en los árboles $\mathrm{C} / \mathrm{C}$ como 
Tabla 1. Microhongos epífitos aislados en corteza-tronco según edad y presencia de cancro

\begin{tabular}{|c|c|c|c|c|c|c|c|c|}
\hline Taxa aislados & \multicolumn{4}{|c|}{ GRUPO 1 (Arboles jovenes) } & \multicolumn{4}{|c|}{ GRUPO 2(Arboles viejos) } \\
\hline $\mathbb{N}^{0}$ de aislamientos & C/canc. & $\%$ & S/canc. & $\%$ & C/canc. & $\%$ & S/canc. & $\%$ \\
\hline Acremonium strictum W. Gams & 16 & 9,9 & 13 & 13,7 & 12 & 9,1 & 8 & 15,3 \\
\hline Acrospeira mirabilis (Muril) And. & - & - & - & - & - & - & 1 & 1,9 \\
\hline Alternaria alternata (Fr.) Keissl. & 10 & 6,2 & 11 & 11,5 & 12 & 9,1 & 10 & 19,2 \\
\hline Anavigra laxa Sutton & 2 & 1,2 & 1 & 1,0 & 1 & 0,7 & - & - \\
\hline Aspergillus flavus Link ex Gray & - & - & - & - & 2 & 1,5 & - & - \\
\hline Aureobasidium pullulans (de Bary)Arnaud & - & - & - & - & 2 & 1,5 & - & - \\
\hline Botrytis cinerea Pers. exNocca \& Balbis & 2 & 1,2 & - & - & 1 & 0,7 & - & - \\
\hline Ceratocystis microspora (Davids)Davids & 16 & 9,9 & 3 & 3,1 & 11 & 8,4 & 2 & 3,8 \\
\hline Ceratocystis brunneo-ciliatum (Math.K) Hunt & - & - & - & - & 1 & 0,7 & - & - \\
\hline Ceratosporella stipitata (Goidan.)Hughes & 1 & 0,6 & 2 & 2,1 & 4 & 3,0 & - & - \\
\hline Chalara unicolor Hughes & 4 & 2,5 & 2 & 2,1 & 4 & 3,0 & 1 & 1,9 \\
\hline Chloridium clavaeforme (Pr.)W.Gams \&Hol.-Jech & 7 & 4,3 & 1 & 1,0 & - & - & - & - \\
\hline Cladosporium cladosporioides (Fres)de Vri. & 3 & 1,8 & 2 & 2,1 & 3 & 2,3 & - & - \\
\hline Chrysosporiumesp. & - & - & - & - & - & - & 1 & 1,9 \\
\hline Coryneum modonium (Tul.) Griff. \& Maubl. & 2 & 1,2 & 9 & 9,5 & 1 & 0,7 & - & - \\
\hline Cryptosporiopsis quercina Petrak & - & - & 1 & 1,0 & - & - & - & - \\
\hline Cryphonectria parasitica (Murr.) Barr & 16 & 9,9 & 4 & 4,2 & 12 & 11,4 & 5 & 9,6 \\
\hline Cylindrocarponsp. & - & - & 1 & 1,0 & - & - & - & - \\
\hline Dactylaria purpurella (Sacc.) Sacc. & 2 & 1,2 & 1 & 1,0 & - & - & - & - \\
\hline Diatrypella quercina (Persoon) Cooke & 1 & 0,6 & - & - & - & - & - & - \\
\hline Dictyochaetasp. & 1 & 0,6 & 1 & 1,0 & - & - & - & - \\
\hline Diplococcium spicatum Grove & 2 & 1,2 & - & - & 3 & 2,3 & - & - \\
\hline $\begin{array}{l}\text { Exochalara longissima (Grove) W.Gams \& } \\
\text { Hol.-Jech. }\end{array}$ & - & - & 1 & 1,0 & 2 & 1,5 & - & - \\
\hline Epicoccum purpurascens Link ex Schlecht & 2 & 1,2 & 6 & 6,3 & - & - & - & - \\
\hline Fusarium moniliforme Link ex Fr. & 6 & 3,7 & 1 & 1,0 & - & - & - & - \\
\hline Gliocladium roseum Bainier & 2 & 1,2 & 1 & 1,0 & 2 & 1,5 & - & - \\
\hline Graphium penicillioides complex Corda & - & - & - & - & 2 & 1,5 & - & - \\
\hline Heteroconium tetracoilum (Corda)M.B.Ellis & 2 & 1.2 & 2 & 2,1 & 1 & 0,7 & - & - \\
\hline Monodictys castaneae (Wallr.) Hughe & 3 & 1,8 & 1 & 1,0 & - & - & - & - \\
\hline Mortierella isabellina Oudem. & 2 & 1,2 & - & - & 3 & 2,3 & 2 & 3,8 \\
\hline Nectria cinnabarina (Tode ex Fr.) Fr. & 1 & 0,6 & - & - & - & - & - & - \\
\hline Nigrospora sphaerica (Sacc.) Mason & 2 & 1,2 & 1 & 1,0 & - & - & - & - \\
\hline Oidiodendron griseum Robak & 2 & 1,2 & - & - & 4 & 3,0 & 2 & 3,8 \\
\hline Ophiostoma stenocerans (Robak)Melin \& Nann. & 3 & 1,8 & - & - & 4 & 3,0 & - & - . \\
\hline Paecilonyces farinosus Bainier & - & - & - & - & 2 & 1,5 & - & - \\
\hline Penicillium spp. & 16 & 9,9 & 16 & 16,8 & 12 & 11,4 & 8 & 15,3 \\
\hline Periconia macrospinosa Lefebvre \& A.G.Johnson & 1 & 0,6 & - & - & - & - & - & - \\
\hline Phialocephala fumrosa (Ellis \& Ev.) Sutton & 4 & 2,5 & - & - & 2 & 1,5 & - & - \\
\hline Phomasp. & 2 & 1,2 & - & - & 2 & 1,5 & - & - \\
\hline Phomopsissp. & - & - & 1 & 1,0 & - & - & - & - \\
\hline Polyscytatum fecundissimum Riess & - & - & - & - & 3 & 2,3 & 1 & 1,9 \\
\hline Pseudopezizasp. & - & - & - & - & 1 & 0,7 & - & - \\
\hline Ramichloridium schulzeri (Sacc.) de Hoog & 4 & 2,5 & 1 & 1,0 & - & - & - & - \\
\hline Rhinocladiella atrovirens Nannf. & 2 & 1,2 & - & - & - & - & - & - \\
\hline Sclerotiniasp. & - & - & - & - & 1 & 0,7 & - & - \\
\hline Spleaeropsis sapinea (Fr.) Dyko \& Sutton & - & - & - & - & 2 & 1,5 & - & - \\
\hline Sporidesmium rubi Ellis & 3 & 1,8 & 7 & 7,4 & 4 & 3,0 & 2 & 3,8 \\
\hline Sporidesmium folliculatum (Corda)Mas.\&Hugh. & 1 & 0,6 & - & - & - & - & - & - \\
\hline Stagonosporasp. & - & - & - & - & 2 & 1,5 & 1 & 1,9 \\
\hline Torula kerbarum (Pers.) Link ex Gray & - & - & - & - & 2 & 1,5 & 2 & 3,8 \\
\hline Trichoderma spp. & 13 & 8,1 & 3 & 3,1 & 8 & 6,1 & 6 & 12,5 \\
\hline Valsa ceratophora Tul. \& C. Tul. & 1 & 0,6 & - & - & - & - & - & - \\
\hline Verticilliumsp. & 2 & 1,2 & 2 & 2,1 & 3 & 2,3 & - & - \\
\hline TOTALES & 161 & & 95 & & 131 & & 52 & \\
\hline
\end{tabular}


Tabla 2. Microhongos epífitos aislados en corteza-base segúm edad y presencia de cancro

\begin{tabular}{|c|c|c|c|c|c|c|c|c|}
\hline \multirow{2}{*}{$\begin{array}{l}\text { Taxa aislados } \\
\qquad \mathrm{N}^{\circ} \text { de aislamientos }\end{array}$} & \multicolumn{4}{|c|}{ GRUPO 1 (Arboles jovenes) } & \multicolumn{4}{|c|}{ GRUPO 2 (Arboles viejos) } \\
\hline & C/canc. & $\%$ & S/canc. & $\%$ & C/canc. & $\%$ & S/canc. & $\%$ \\
\hline Acremonium strictum W. Gams & 9 & 8,0 & 12 & 12,2 & 7 & 8,6 & 7 & 14 \\
\hline $\begin{array}{l}\text { Acrodontium anam.Ascocorticium anoma- } \\
\text { lum (Ellis \& Harkn.) Earle }\end{array}$ & - & - & 1 & 1,0 & - & - & - & - \\
\hline Alternaria alternata (Fr.)Keissl. & 1 & 0,8 & 1 & 1,0 & 1 & 1,2 & 1 & 2,0 \\
\hline Anthostomella fuegiana Sacc. & 1 & 0,8 & 2 & 2,0 & - & - & - & - \\
\hline Botrytis cinerea Pers. exNocca \& Balbis & - & - & - & - & 1 & 1,2 & - & - \\
\hline Calonectriasp. & - & - & 1 & 1,0 & - & - & - & - \\
\hline Ceratocystis microspora (Davids)Davids & 4 & 3,6 & 3 & 3,0 & 3 & 3,7 & 1 & 2,0 \\
\hline Ceratocystisbrunneo-ciliatum (Math.K)Hunt & - & - & 1 & 1,0 & - & - & - & - \\
\hline Ceratosporella stipitata (Goidan.)Hughes & - & - & - & - & - & - & 1 & 2,0 \\
\hline Chalara unicolor Hughes & 5 & 4,5 & 8 & 8,2 & 6 & 7,4 & 3 & 6,0 \\
\hline Chloridium clavaeforme (Pre.)WGams \&Hol.-Jech & 6 & 5,3 & 5 & 5,1 & 7 & 8,6 & 3 & 6,0 \\
\hline Cladosporium cladosporioides (Fres.) de Vri.. & 1 & 0,8 & 2 & 2,0 & - & - & 1 & 2 \\
\hline Codinea britannica M.B.Ellis & 2 & 1,9 & 1 & 1,0 & - & - & - & - \\
\hline Coniella castaenicola (Ellis.\& Ev.) Sutton & 1 & 0,8 & - & - & 1 & 1,2 & - & - \\
\hline Corniculariella spina (Berk. \& Rav.)di Cosmo & - & - & 1 & 1,0 & - & - & - & - \\
\hline Cryphonectria parasitica (Murr.) Barr & 7 & 6,2 & 2 & 2,0 & 9 & 11,1 & 2 & 4,0 \\
\hline Cryptosporiopsis quercina Petrak & 1 & 0,8 & - & - & - & - & - & - \\
\hline Dactylaria purpurella (Sacc.) Sacc. & 3 & 2,7 & 2 & 2,0 & 1 & 1,2 & 3 & 3,0 \\
\hline Dactylellabembicodes Drechsler & 4 & 3,6 & 3 & 3,0 & 1 & 1,2 & - & - \\
\hline Dinemasporium strigosum (Pers ex Fr)Sacc. & - & - & 1 & 1,0 & - & - & - & - \\
\hline Diplococcium spicatum Grove & 3 & 2,7 & 2 & 2,0 & 2 & 2,5 & 1 & 2,0 \\
\hline Diplococcium ana.Helminth.clav:(Tul.)Fuck. & 1 & 0,8 & 1 & 1,0 & - & - & - & - \\
\hline Endophragmia hialosperma (Cor)M.Jones-\&Cole & - & - & 1 & 1,0 & - & - & - & - \\
\hline Fusarium moniliforme Link ex Fr. & 2 & 1,9 & - & - & - & - & - & - \\
\hline Gliocladium roseum Bainier & 1 & 0,8 & - & - & - & - & 1 & 2,0 \\
\hline Gonytrichum caesium C.G \& F. Nees var.caesium & - & - & - & - & 2 & 2,5 & - & - \\
\hline Heteroconium tetracoilum (Corda)M.B.Ellis & - & - & - & - & 1 & 1,2 & - & - \\
\hline Hypoxylon mediterraneum (DeNot..)Ces \& DNot & 1 & 0,8 & - & - & - & - & - & - \\
\hline Libertella faginea Des. & 1 & 0,8 & - & - & - & - & - & - \\
\hline Memmoniella echinata (Riv.) Galloway & - & - & - & - & - & - & 1 & 2,0 \\
\hline Monodictys castaneae (Wallr.) Hughes & 3 & 2,7 & 3 & 3,0 & 1 & 1,2 & - & - \\
\hline Mortierella isabellina Oudem & 6 & 5,3 & 7 & 7,1 & 4 & 4,9 & 3 & 6,0 \\
\hline Mortierella ramanniana (Möller)Linn. var raman. & 1 & 0,8 & - & - & 1 & 1,2 & - & - \\
\hline Oidiodendron griseum Robak & 5 & 4,5 & 6 & 6,1 & 3 & 3,7 & 4 & 8,0 \\
\hline Oncopodiella trigoniella (Sacc.) Rifai & - & - & 1 & 1.0 & - & - & - & - \\
\hline Opltiostoma stenocerans (Rob.)Melin \& Nann. & 2 & 1,9 & - & - & 1 & 1.2 & 1 & 2.0 \\
\hline Paecilomyces farinosus Bainier & 1 & 0,8 & 1 & 1,0 & 2. & 2,5 & 1 & 2,0 \\
\hline Penicillium spp. & 11 & 9,8 & 9 & 9,2 & 8 & 9,9 & 5 & 10,0 \\
\hline Phialocephala fumosa (Ellis \& Ev.) Sutton & 5 & 4,5 & 4 & 4,0 & 3 & 3,7 & 1 & 2,0 \\
\hline Pilidium acerinum Kunze apud Kun.\& Schm. & 2 & 1,9 & - & - & 2 & 2,5 & - & - \\
\hline Phomasp. & - & - & - & - & - & - & 1 & 2,0 \\
\hline Polyscytatum fecundissimum Riess & 3 & 2,7 & 4 & 4,0 & - & - & 2 & 4.0 \\
\hline Pseudodiplodia lignaria (Karst.) Sacc. & 1 & 0,8 & - & - & - & - & - & - \\
\hline Pseudogymnoascus roseus Raillo & - & - & $1-$ & - & - & - & 1 & 2,0 \\
\hline Ramichloridium schulzeri (Sacc.) de Hoog & - & - & 2 & 2,0 & - & - & - & - \\
\hline Rhinocladiella atrovirens Nannf. & 3 & 2,7 & 1 & 1,0 & - & - & - & - \\
\hline Rhizopus stolonifer (Ehremb ex Link) Lind & 1 & 0,8 & 1 & 1,0 & & & & \\
\hline Septonemasecedens Corda & - & - & 1 & 1,0 & - & - & - & - \\
\hline Spluaeropsis sapinea (Fr.) Dyko \& Sutton & - & - & - & - & 2 & 2,5 & - & - \\
\hline Sporidesmium rubi Ellis & 4 & 3,6 & 2 & 2,0 & 3 & 3,7 & 3 & 6,0 \\
\hline Taenionella stilbospora (Corda) Hughes & - & - & - & - & 2 & 2,5 & - & - \\
\hline Trichoderma spp. & 4 & 3,6 & 3 & 3,0 & 6 & 7,4 & 1 & 2,0 \\
\hline Verticillium spp. & 2 & 1,9 & 2 & 2,0 & 1 & 1,2 & 2 & 4,0 \\
\hline Verticilliumtenuissimum Corda & 4 & 3,6 & - & - & - & - & - & - \\
\hline Verticillium psallotiae Treschow & - & - & 1 & 1.0 & - & - & - & - \\
\hline TOTALES & 112 & & 98 & & 81 & & 50 & \\
\hline
\end{tabular}


GRAFICO 1. AISLAMIENTOS DE MICROHONGOS EPIFITOS SEGUN TIPO DE CORTEZA, EDAD Y PRESENCIA DE CANCRO

$$
N^{\circ}
$$

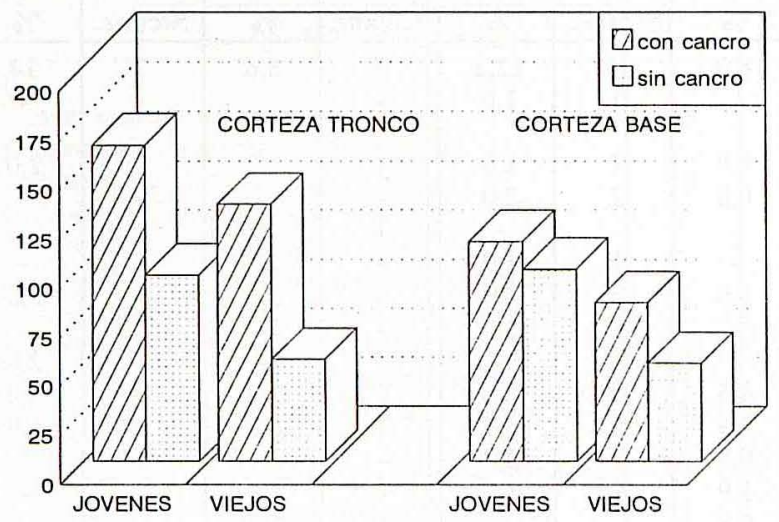

GRAFICO 2. TAXA DE MICROHONGOS EPIFITOS SEGUN TIPO DE CORTEZA, EDAD Y PRESENCIA DE CANCRO.
GRAFICO 3. MICROHONGOS EPIFITOS, PATOGENOS U OPORTUNISTAS SEGUN TIPO DE CORTEZA, EDAD Y PRESENCIA DE CANCRO.

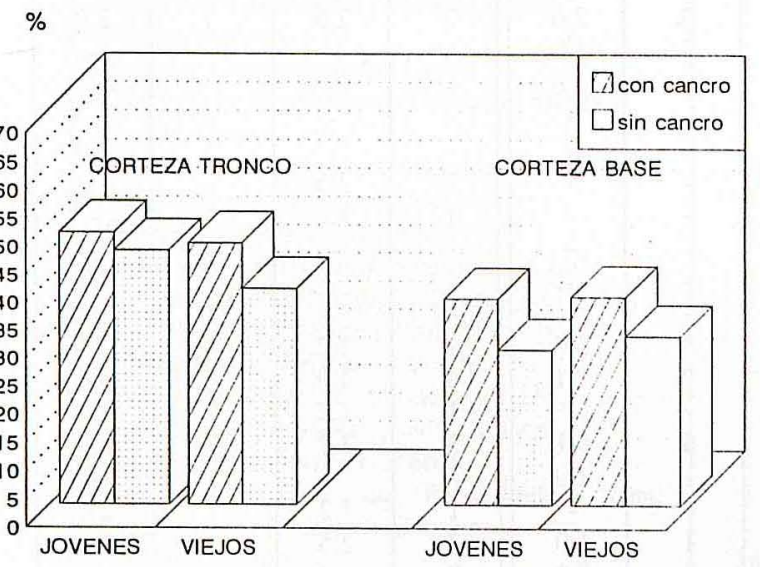

en $\operatorname{los} \mathrm{S} / \mathrm{C}$, en todas las situaciones. En la condición donde mejor se presenta este pool, es en $\mathrm{C} / \mathrm{T}$ con cancro ya sea en arboles jóvenes o viejos. En los restantes, algunas de ellas o ambas, son disminuidas en sus proporciones por la presencia de Coryneum modonium o Chalara unicolor. Mientras la colonización de $C$.unicolor aumenta en la $\mathrm{C} / \mathrm{B}$, en especial en los arboles S/C (Gráfico 4).

Sólo $8(10,5 \%)$ del total de los 78 taxa detectados, se presentaron en todas las combinaciones de sustratos estudiados, estos fueron: Acremonium strictum, Alternaria alternata, Ceratocystis microspora, Cryphonectria parasitica, Penicillium spp., Sporidesmium rubi y Trichoderrma spp. (Tabla 1 y 2 ).
No

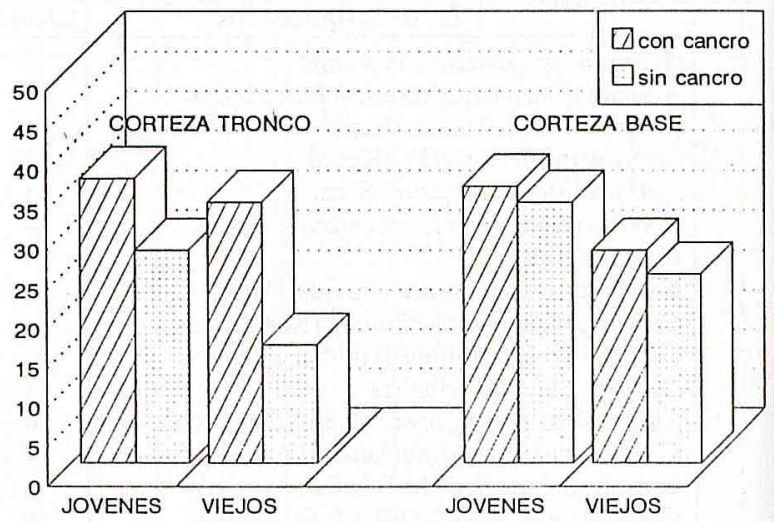

AFICO 4. PRINCIPALES MICROHONGOS PATOGENOS U OPORTUNISTAS SEGUN TIPO DE CORTEZA, EDAD Y PRESENCIA DE CANCRO.

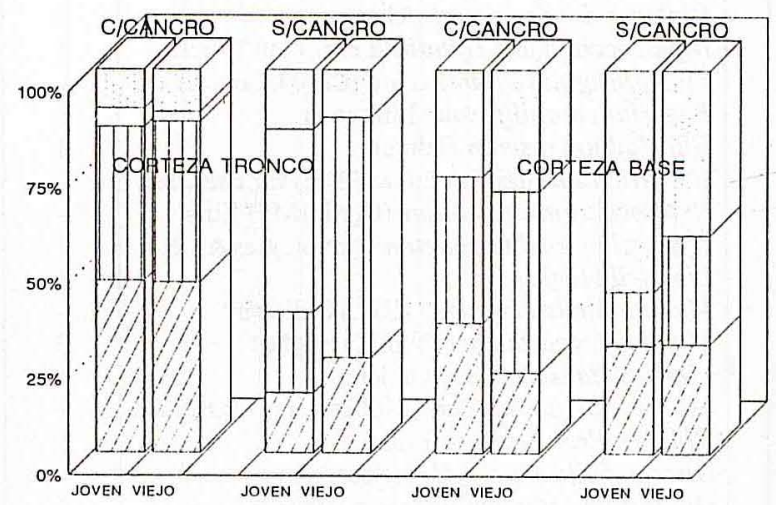

ঢCerat-Ophi. []

Las más altas diversidades (Shannon-Wieber), se observaron en árboles con cancro (sobre 2,9). La diversidad es siempre mayor en los árboles jovenes que en los viejos y es más variable en C/B (de 2.10 a 3.3) que en $\mathrm{C} / \mathrm{T}$ (de 2.4 a 3) (Gráfico 5).Las similitudes de Jaccardmás altas, se observaron entre árboles jovenes con y sin cancro y entre $\mathrm{J}$ y V( $\mathrm{C} / \mathrm{C})$, que son precisamente los que presentaron las mayores diversidades. Las mayores similitudes se observan entre los árboles sin cancro en $\mathrm{C} / \mathrm{T}$ y entre $\mathrm{C} / \mathrm{T}$ y $\mathrm{C} / \mathrm{B}$ (Gráfico 5.)

\section{a) Especies en la corteza tronco.}

Los taxa principales predominantes ya sea en los gruposdeárboles Jo V (C/C) fueron :Acremonium strictum, 
Alternaria alternata, Ceratocystis microspora, Cryphonectria parasitica, Penicillium spp. y Trichoderma spp, con bastante similitud en presencia y porcentajes ( 53,9 a 55,5\% de ocurrencia del total de la micota presente). En los árboles $\mathrm{S} / \mathrm{C}$, hubo diferencias en porcentajes y en presencia, en los jovebnes: $A$ strictum, $A$. alternata, Coryneum modonium, Epicoccum purpurascens, Penicillium spp. y Sporidesmium rubi $(65,2 \%$ del total de la micota), mientras en los viejos, fueron predominantes: $A$. strictum, $A$. alternata, Cr.parasitica, Penicillium spp. y Trichodrerma spp. representandoel 71,9\% del total (Tabla 1).

b) Especies en la corteza base.

Los principales taxa predominantes en los árboles $\mathrm{J}$ (C/C) fueron: A. strictum, Chalara unicolor, Chloridium clavaeforme, Cr. parasitica, Penicilliumn spp. (con un 40,1\% del total), mientras en los V : A. strictum, C. unicolor, C. clavaeforme, Cr. parasitica, Penicillium spp . y Trichoderma spp, con un $53 \%$ del total de la micota. En los J (S/C): A. strictum, Ch. unicolor, Ch.clavaeforme, Mortierella isabellina, Oidiodendron griseum y Penicillium spp. con un $47.9 \%$ del total, mientras en los V (S/C) se detectaron las mismas especies además de Sporidesmium rubi con un $44 \%$ del total de la micota (Tabla 2).

\section{Gráfico 5.}

Similitud de Jaccard y Diversidad de Shannon Wieber según grupos y categorías

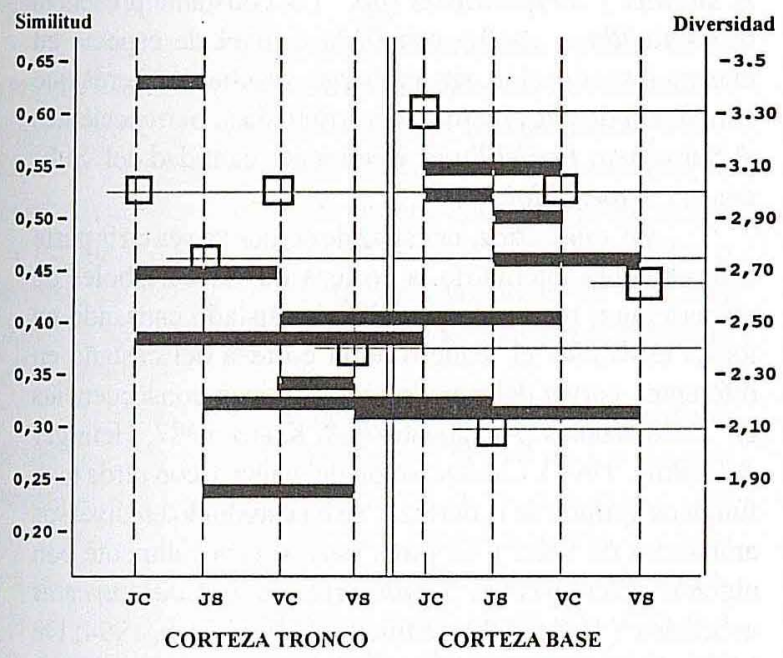

$\mathrm{JC}=$ Arboles jovenes con cancro J8 = Arboles jovenes sin cancro $\nabla C=$ Arboles viejos con cancro $\quad \nabla 8=$ Arboles viejos $\sin$ cancro

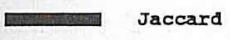

Shannon-wieber

\section{DISCUSION}

Nuestros resultados han permitido obtener un cuadro general de un grupo de especies, capaces de crecer sobre la corteza del tronco y el cuello del castaño, ante la presencia o ausencia de cancro, en un grupo etáreo de una región del norte de Italia. Estos microhongos a pesar de no ser en su totalidad de un notable interés fitopatológico, lo son bajo el aspecto de la biodiversidad fúngica, ya sea por la escasa distribución de algunos en este biotopo, o a la particular asociación que otros representan con la corteza de Castanea sativa en diversas localidades geográficas (Bassi,1990; Ellis \& Ellis,1985; Cobos, 1989; Anognostakis, 1990; Baird, 1991; Conedera, 1991)

A pesar que en los tejidos de la corteza de los árboles vivos, puede existir una gran variedad de organismos, los hongos son siempre mayoritarios y en especial los Duteromycetes (Cotter \& Blanchard, 1982,Baird, 1991).En nuestro estudio, la mayor parte pertenecieron a este grupo y dentro de éstos, los dematiaceos presentaron gran diversificación. En menor número, varios representantes de los Ascomycota también se asociaron a la corteza y solo 2 especies del género Mortierella y una del género Rhizopus, fueron los representantes de los Zycomycetes.

La mayor cantidad de aislamientos correspondió siempre en todos los tipos de sustrato, a los árboles más jovenes $(\mathrm{C} / \mathrm{C})$, lo cual puede atribuirse al estress y debilitamiento causado por Cr.parasitica, mientras en la $\mathrm{C} / \mathrm{B}$ de los árboles viejos $\mathrm{S} / \mathrm{C}$, la mayor diversidad de especie, puede relacionarse en parte a las influencias de la microbiota del suelo y de la hojarasca circundante, situación que se aprecia por el aumento de ciertas especies pertenecientes a los géneros Morteriella, Chalara, Chloridium, Phialocephala, entre otras.

La presencia fúngica en ambos tipos de corteza, no solo puede relacionarse a este particular sustrato, sino a la presencia de: tejidos necróticos (abundantes en los castaños $\mathrm{C} / \mathrm{C}$ ), aptos para la colonización de patógenos necrotrofos, la influencia de factores climáticos-ambientales (dispersión anemófila, el contenido de humedad, $\mathrm{pH}$, temperatura, etc.), la presencia de exudados, microorganismos e insectos rastreros y volátiles. El contenido de humedad afecta significativamente la colonización fúngica, en especial se obseva mayor abundancia con contenidos más bajos de agua que en los más altos, situación que permite considerar el mejor método de prevención para la invasión de la madera por hongos patógenos o degradadores (Rayner \& Boddy, 1986, Chapela \& Boddy, 1988). Esta situación tambien fue osbservada en los hongos endófitos de Castanea sativa por Bissegger \& Sieber (1994).

Los insectos (Coleopteros y Acaros principalmente), son habitantes comunes o de paso, capaces de trains- 
portar propágulos fúngicos a distancia y por ende, reunen características de vectores asociados a la transmisión de enfermedades fúngicas del castaño (Turchetti \& Chelazzi, 1984; Russin et al., 1984; Nanelli \& Turchetti, 1989).

La abundancia de insectos, sus restos y sus excretas, aportan constantemente nuevos sustratos fres$\cos$ ricos en proteinas, aptos para el desarrollo de una micobiota particular mayoritariamente saprotrofa, que muestra sin duda, dos características principales: elevada selectividad específica por el tejido o elevada adaptación inespecífica por los sustratos heterogéneos asociados a la corteza. Ambas situaciones, parecen facilitar no solo el desarrollo de una comunidad típica del castaño, como Monodictys castaneae, Ceratocystis microspora, Cryphonectria parasitica, Coryneum modonium, Valsa ceratophora, u otras, sino de otras consideradas específicas de los involucros (erizos), frutos y hojas de este árbol, como es el caso de Acrospeira mirabilis, Anavigra laxa (solo presente en $\mathrm{C} / \mathrm{T}$ ), Chalara spp. (en nuestro caso Chalara unicolor), Codinea britannica, Pilidium acerinum, Polyscytatum fecundissimum. Phialocephala fumosa, entre otros. Esta situación permite descartar en cierta medida que la corteza de cada árbol tiene una particular especificidad (Greenhalgh, 1969). Más bien parece indicar que parte de la comunidad epífita es inespecífica(entre un 30 a 40\%), principalmente Hyphomycetes y algunos Coelomycetes y pueden ser capaces de colonizar indiferentemente el mismo sustrato $\mathrm{u}$ otro, en varias especies de árboles de madera dura, en zonas geográficas que reunan condiciones climáticas semejantes, comolo afirman Cotter \& Blanchard (1982) y Baird(1991).

Varios estudios confirman que la densidad de colonización de los epífitos en la corteza es mucho más alta que los endófitos capaces de infectar tejidos profundos o el xilema y que estos últimos producen su infección, después que han colonizado extensivamente la corteza (Boddy \& Griffith, 1989; Petrini \& Fisher, 1990).

A pesar que existen ciertas diferencias entre las comunidades de los árboles $\mathrm{C} / \mathrm{C}$ y los $\mathrm{S} / \mathrm{C}$, esto sugiere que la mayoria, son habitantes de la corteza y su presencia no interfiere con la de determinados patógenos (Baird,1991). Este último autor, en West Virginia (U.S.A.), encontró una comunidad de 35 géneros de microhongos sobre la corteza de Castanea dentata, previamente inoculada con Cr. parasitica (cepas hipovirulentas). E160\% de éstos (21 géneros), fueron aislados también en nuestra investigación. De los 25 taxa de Asco-Deuteromycetes aislados por Cobos (1989), en Castanea sativa, en España, el 60\% fue igual a nuestros aislamientos y su revisión de la literatura internacional detectada sobre Castanea, contiene un $65 \%$ de los géneros de Hyphomycetes registrados en nuestro trabajo.

La dominancia de algunas especies, creciendo en proximidad, puede representar un importante factor antagónico que puede interferir ya sea en: la estimulación trófica, las alteraciones morfogenéticas, la inhibición del crecimiento y el grado de patogénesis de algunos biotrofos u oportunistas fúngicos de árboles. La interacción hifal, es considerada importante en la determinación del patron decolonización de los excrementos (Ikediugwu \& Webster, 1970), como asi la competencia (antagonismo) entre especies de Trichoderma y otros hongos (Nelson, 1982; Biles\&Hill,1988).

Ennuestros aislamientos de Trichoderma,T.viride, fue la especie más común y llama la atención que todos los representantes del género fueron mayoritariamente aislados de los árboles $\mathrm{C} / \mathrm{C}$, en ambos sustratos. Esto podría indicar un escaso antagonismo con los epífitos dominantes o los endófitos presentes en los castaños estudiados, como: Cr. parasítica ( y Endothiella parasitica su anamorfo, observada varia veces junto a su teleomorfo), Coniella castaneicola, Coryneummodonium, Phomopsis $\boldsymbol{s p}$. Estas mismas especies y otras fueron también detectadas por Bissegger \& Sieber,(1994) en el sur y norte de los Alpes suizos.

De Martino et al. (1994), estudiaron el antagonismo de algunos endófitos del castaño, pudiendo observar un cierto grado de actividad inhibitoria de Coryneum modonium hacia Cr. parasitica, mientras Minervini \& Bisiach (1994), en una localidad geográficamente cercana a nuestra zona de muestreo, encontraron actividad inhibitoria in vitro de algunos epífitos comunes de la corteza hacia $C r$. parasitica, como: Penicillium expansum $\boldsymbol{P}$. janczewskii, Chaetomium globosum, Bacillus polymixa, B. subtilis y Streptomyces spp. La constante presencia de Penicillium, no fue estudiada a nivel de especie en nuestra investigación, sin embargo resulta de interés que la mayoría de ellas fueron terverticiladas, pertenecientes al Subgénero Penicillium y en menor cantidad del Subgénero Aspergilloides.

Cr. parasitica, es capáz de crecer ya sea en la parte externa como interna de la corteza de varios árboles de madera dura, pero en especial se ha aislado causando en forma específica el cancro de la corteza del castaño en diferentes partes del mundo, con trágicas consecuencias en estós árboles (Anagnostakis \& Kranz 1987; Heiniger \& Rigling, 1994). La asociación del cancro con otros taxa fúngicos epífitos de la corteza, se ha estudiado en diversos ambientes de Italia y en otros paises, especialmente con algunos Ascomycetes, Hyphomycetes y Coelomycetes asociados (Baird, 1991; Minervini \& Bisiach, 1994; De Martino et al., 1994), en especial con Ceratocystis microspora (Davidson \& Kuhlman, 1978; Russin \& Shain, 1984). Esto último se manifestó también en el grupo de nuestros castaños, en los árboles $\mathrm{C} / \mathrm{C}$ y S/C y en menor porcentaje también asociaciada a otra especie relacionada, 
como Ophiostoma stenocerans (=C. eucastaneae, fide Upadhyay, 1981) y su anamorfo morfológicamente similar a Sporothrix schenkii (S.pallida o S. nivea, De Hoog, 1993,Fig24). Según Russin \& Shain (1984), C. microspora se establece anteriormente a $\boldsymbol{C r}$. parasitica en el cancro del castaño y es estimulada en su crecimiento por los metabolitos de esta última, situación que observamos en la corteza in vitro, como un constante incremento de los peritecios de $C$. microspora y $\boldsymbol{O}$. stenocerans en el tiempo.

Es necesario también destacar que sobre Cr.parasitica se desarrolló y asoció frecuentemente Acremonium strictum, un taxa común del suelo y otros habitat y no es raro observarlo sobre royas y oidios. Una situación similar pudo detectarse entre $C$. microspora y la constante colonización de sus cuellos por Phialocephala fumosa.

En Italia, la aparición de cepas hipovirulentas naturales de Cr.parasútica, capaces de transferir por anastomosis de sus hifas, partículas virales de ARN de doble cadena a las cepas hipervirulentas, para tranformarlas en hipovirulentas, ha permitido un control biológico natural y la recuperación del castaño con poca intervención humana . Sin embargo, la inoculación de cepas europeas hipovirulentas en U.S.A, no terminaron con la enfermedad y el castaño americano (Castanea dentata) no ha llegado al estado adulto en las regiones donde $\boldsymbol{C r}$. parasitica es endémica, debido a un pool de genes que regulan la compatibilidad vegetativa de sus hifas (Anagnostakis, 1980, 1987). Esta hipovirulencia transmisible, asegura un modelo para el control de esta enfermedad y la movilidad de elementos citoplasmáticos en las poblaciones de Hyphomycetes.

Podemos concluir que la microbiota epífita de la corteza del castaño fluctua en relación a factores ambientales, geográficos y nutricionales. Las características topográficas y la heterogeneidad de los sustratos presentes en diferentes partes de la corteza, puede aumentar la eficiencia de la colonización de ciertas especies residentes, epífitas o endófitas, permitiendo una mayor diversidad, en especial en los árboles $\mathrm{C} / \mathrm{C}$, quizás debido a la acción preparatoria de Cr. parasitica.

A pesar que no hemos estudiado su presencia estacional en el año, el crecimiento activo de los hongos detectados sobre este sustrato nos lleva a suponer que corresponden mayoritariamente a especies residentes de la corteza y estas últimas junto a las alóctonas, pueden prevenir la invasión de patógenos y ejercer en determinados momentos de sus ciclos de vida, efectos inhibitorios que merecen estudiarse como posibles agentes naturales de biocontrol.

La comunidad fúngica de ambos tipos de corteza parece estar compuesta por un pequeño grupo de especies dominantes, ya sea epífitas como endófitas, con ciertas diferencias en la composición de sus especies.
Como las mayores diversidades se observaron siempre en los árboles $\mathrm{C} / \mathrm{C}$ en ambos tipos de sustratos, esto sugiere, que muchas especies frente a una respuesta disminuida del hospedador actuarían como oportunistas. Como no se determinó la alta actividad de laccasa de las cepas de Cr.parasitica, en los árboles $\mathrm{C} / \mathrm{C}$ y $\mathrm{S} / \mathrm{C}$, sus fenotipos pueden ser normales, hipovirulentos $O$ corresponder a estados de latencia (en los árboles S/C aparente), una situación observada por Russin \& Shain (1984). Esto puede atribuirse a una resistencia de los castaños, a la presencia de cepas hipovirulentas (una situación que debe considerarse), o simplemente a un tiempo de latencia largo (común en los endófitos), en espera de los momentos de estress del hospedador.

En nuestro trabajo exploratorio, un buen porcentaje de las especies detectadas y que presentan una marcada preferencia por la corteza de este árbol, no han sido reportadas en Italia.

\section{REFERENCIAS}

Anagnostakis,S.L. (1980). Notes on the genetics of Endothia parasitica. Neurospora Newsletter 27:36

Anognostakis,s.L. (1987). Chestnut Bligh: The classical problem of an introduced pathogen. Mycologia 79:23-37

Anognostakis, S.L. (1990). An historical reference for chestnut introductions in North America. Annual Report- Nort-hern Nut Growers' Associatin $N^{\circ} 80: 132-143$

Anognostakis,S.L. \& Kranz,J. (1987). Population dynamics of Chryphonectria parasitica in a mixed-hardwood forest in Connecticut. Phytopathology 77:751-754

Baird,R.E. (1991). Mycobiota of bark associated with se-ven strains of Cryphonectria parasitica on two hardwood tree species. Mycotaxon 40:23-33

Bassi,R. (1990). La coltivazione del castagno. Ed. L'informatore agrario, Roma.

Behrend,C.J.; Blanchette,R.A.; Farrel,R.L. (1994). Biological control of Blue-stain Fungi in Wood. Phytopathology 85:92-97

Biles,C.L \& Hill,J.P. (1988). Effect of Trichoderma harzianum on sporulation of Cochliobolus sativus on excised wheat seedling leaves. Phytopathology 78: 656-659

Biraghi.A. (1950). La distribuzione del cancro del castagno in Italia. L'Italia Forestale e Montana 5:18-21

Bisseger, M. \& Sieber, T.N. (1994). Assemblage of endophytic fungi in coppice shoots of Castanea sativa. Mycologia 86:648-655

Boddy,L. \& Griffith,G.S.(1989). Role of endophytes and latent invasion in the development of decay communities in sapwood of angiospermous trees. Sydowia 41:41-73

Chapela,I.H. \& Boddy,L. (1988). Fungal colonization of attached beech branches.II. Spatial and temporal organization of communities arising from latent invaders in bark and functional sapwood under different moisture regimes. New Phytol. 110:47-57

Cobos,S.P. (1989). Fitopatologìa del castaño (Castanea sativa Miller). Boletìn de Sanidad Vegetal( Fuera de serie) $N^{\circ} 16$. Secretarì general Tècnica, Ministerio de Agricultura, Pesca y Alimentaciòn, Madrid. 
Conedera,M. (1991). La situazione del cancro corticale del castagno (Chryphonectria parasitica (Murr.) Barr, al sud delle Alpi (Svizzera meridionale). Schweizerische Zeitschrift fur Forstwesen 142:283-298

Cotter,H.Van T. \& Blanchard, R.O. (1982). The fungal flora of bark of Fagus grandifolia. . Mycologia 74:836-843

De Martino, A.; Intropido, M.; Bisiach,M. (1994) Chestnut decline caused by Diplodina castaneae and Coryneum modonium.

Atti Intern Congress on Chestnut. Informatore Fitopatologico 5: 26-28.

Davidson,R.W.\& Kuhlman,E.G. (1978).A species of Ceratocystis closely associated with Endothia cankers on American chestnut of eastern United States. Mycologia 70:853-855

Ellis,M.B. \& Ellis,J.P. (1985). Microfungi on land plants an identification handbook. Crom Helm, London \& Sydney

Goidanich,G. (1960). L'avversita delle piante agrarie. Ramo editoriale degli agricoltori, Roma 2 :97-104

Gobbi,E. \& Locci,R. (1989). Aspetti micologici e patologici di Chryphonectria parasitica. Micologia Italiana 18:111/31-111/36

Greenhalgh,G.N. (1969). The ecology of bark inhabiting fungy (Abstract written by M. Holden.) Bull. Brit. Mycol. Soc. 3:82

Heiniger, U. \& Rigling,D. (1994). Biological control of Chestnut blight in Europe. Annual Rev. Phytopathol. 32: 118-142

Hepting,G.H. (1974). Death of American chestnut. Jour-nal Forest History 18: 60-67

Hoog, G.S. de. (1993) Sporothrix- - like anamorphs of Ophiostoma species and other fungi. In : Wingfield J.M. et al. Ceratocystis and Ophiostoma Taxonomy, Ecology and Pathogenicity.APS Press. St.Paul. Minnesota. pp. 53-60

Ikediugwu, F.E.O. \& Webster, J. (1970). Hyphal interference in a range of coprophilous fungi. Trans. Br. micol. Soc. 54:205-210

Mansilla,J.P. (1984). Algunos insectos del castaño en Galicia. Congreso internacional sobre el castaño.Lourizan, Pontevedra, pp. 227-237

Minervini,G. \& Bisiach,M. (1994). Interaction between hypovirulent strains of Chryphonectria parasitica and chestnut ecosystem microorganisms. Atti Intern. Congress on Chestnut. Informatore Fitopatologico 5:23-25

Muñoz,M.C \& Cobos,P. (1991). Endothia parasitica (Murril) Anderson, Sintomatologìa e identificaciòn. Situaciòn de la enfermedad en los castañares asturianos. Boletin de Sanidad
Vegetal,Plagas 17:297-298

Nannelli,R. \& Turchetti,T.(1989). Osservazioni preliminari sull' associazione di alcune specie di Acari corticicoli con Cryphonectria parasitica(Murr.) Barr. Redia 72:581-593

Nelson,E.E.(1982). Occurrence of trichoderma in a Douglas-fir soil. Mycologia 74:280-284

Petrini,O.\& Fisher,P. (1990). Occurrence of fungal endophytes in twigs of Salix fragilis and Quercus robur. Mycol. Res. 94:10771080

Rayner, A.D.M. \& Boddy, L. (1986). Population structure and infection biology of wood decay fungy in living trees. Advances PI. Pathol. 5: 119-160

Russin,J.S. \& Shain,L. (1984).Colonization of chestnut blight cankers by Ceratocystis microspora and $C$. encastaneae. Phytopathology 74: 1257-1261

Russin,J.S.; Shain,L.; Nordin,G.L.(1984). Insects as carriers of virulent and cytoplasmic hipovirulent isoaltes of the chestnut blight fungus. J.of Economic Entomol. 77:838-846

Turchetti,T. (1986). Alcuni aspetti delle principali malattie crittogamiche del castagno. Informatore Agrario 42: 51-53

Turchetti,T.\& Chelazzi,G. (1984). Possible role of slugs as vectors of the chestun blight fungus. European J. of Forest Pathol. 14: $125-127$

Turchetti,T.; Maresi,G. (1991). Inoculation trials with hypovirulent strains of Cryphonectria parasitica.. European Journal of Forest Pathology 21:65-70

Turchetti,T; Sottovia,A.; Maresi,G.; Minerbi,S. (1992). Il cancro della corteccia del castagno in Alto Adige. Informatore Fitopatologico 42:45-48

Sung,J. \& Han,S.S. (1986). Identification of canker-causing fungi associated with stem and twigs of chestnut tree.

Korean Journal of Plant Pathology 2:174-184

Upadhyay,H.P.(1981). A monograph of Ceratocystis and Ceratocystiopsis. Unyversity of Georgia Press. Athens, Georgia

Wells,J.M. \& Payne, J. A. (1975). Toxigenic Aspergillus and Penicillium isolates from weevil damaged chestnuts. Applied Microbiology $30: 536-540$

Wilhelm,E. (1992). Use of endophytic bacteria as biocontrol agents against chesnut blight. Proc. Intern. Chestut Conference, Morgantown. West Virginia. pp. 5.

Figuras.- 1. 2.- Gonytrichum caesium var.caesium (anamorfo de Chaetosphaeria inaequalis), complejo sistema de ramificación y conidios (Barra $5 \mu \mathrm{m}$ ). 2.-Células conidiógenas terminales con collarete terminal (Barra $5 \mu \mathrm{m}$ ). 3.-Phialocephala fumosa, conidió foros y conidios cilíndricos(Barra $5 \mu \mathrm{m}$ ). 5.-Verticillium tenuissimum, conidióforo, fiálides y conidios(Barra $15 \mu \mathrm{m})$. 5.-Rhinocladiella atrovirens, conidióforos y conidios (Barra $5 \mu \mathrm{m}$ ). 6.- Cryptosporiopsis quercina, conidios(Barra $20 \mu \mathrm{n}$ ). 7.-Dictyochaeta sp., conidios y conidióforo (Barra $10 \mu \mathrm{m}$ ). 8.- Exochalara longissima, conidióforo y cadenas de conidios (Barra $10 \mu \mathrm{m})$. 9.- Pilidium acerinum, conidios y conidióforos en sección vertical del conidioma (Barra $10 \mu \mathrm{m})$. 10.-Endothiella anamorfo de Cryphonectria parasitica, conidios y conidióforos en sección vertical del conidioma (Barra $5 \mu \mathrm{m}$ ). 11.-Sphaeropsis sapinea, conidios (Barra $20 \mu \mathrm{m})$. 12.Septonema secedens, conidios en cadena (Barra $10 \mu \mathrm{m}$ ). 13.-Coryneum modonium, conidios yracimos de células conidiógenas(Barra $20 \mu \mathrm{m})$. 14. - Chalara unicolor, artroconidios con 3 septos y células conidiógenas(Barra $30 \mu \mathrm{m}$ ). 15.-Oncopodiella trigonella, conidios (Barra $10 \mu \mathrm{m}$ ). 16.- Diplococcium spicatum, conidióforos y conidios (Barra $10 \mu \mathrm{m}$ ). 17.- Chloridium clavaeforme, proliferación percurrente y conidios (Barra $5 \mu \mathrm{m}$ ), 18.-Ceratosporella stipitata, conidio y conidióforo (Barra $10 \mu \mathrm{m})$. 19.-Monodyctis castaneae, conidios (Barra $20 \mu \mathrm{m}$ ). 20.-Sporidesmium rubi, conidio y conidióforo(Barra $10 \mu \mathrm{m}$ ). 21.-Anavigra laxa, stauroconidios (Barra 20 $\mu \mathrm{m})$. 22.-Ceratocystis brunneo-ciliata (?), ascosporas e hifas ostiolares espiraladas (Barra $10 \mu \mathrm{m})$. 23.- Ceratocystis microspora, ascosporas y porción del cuello del peritecio (Barra $10 \mu \mathrm{m}$ ). 24.-Sporothrix anamorfo de Ophiostoma stenocerans, creciendo sobre el cuello del peritecio(Barra $10 \mu \mathrm{m}$ ). 25.-Ophiostoma stenocerans (=Ceratocystis eucastaneae), ascosporas sin vaina (Barra $5 \mu \mathrm{m})$. 26.- Anthostomella fuegiana, ascosporas bicelulares (Barra $10 \mu \mathrm{m}$ ). 

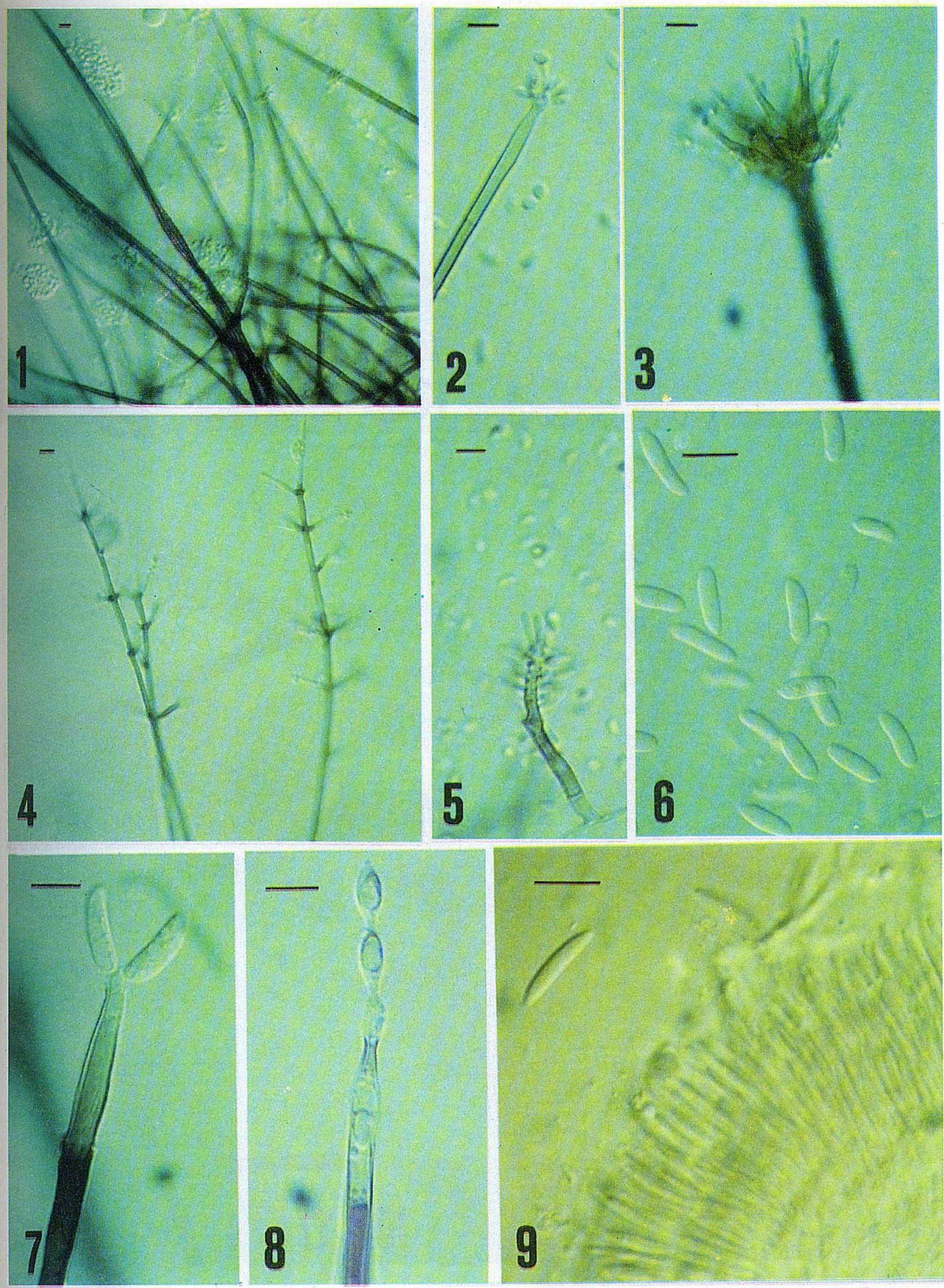